\title{
Global burden of cardiovascular disease
}

The Global Burden of Disease Study 2010, published in seven articles in the Lancet, "is the largest ever systematic effort to describe the global distribution and causes of a wide array of major diseases". The executive summary goes on to state that "infectious diseases, maternal and child illness, and malnutrition now cause fewer deaths and less illness than they did 20 years ago ... as noncommunicable diseases, such as cancer and heart disease, become the dominant causes of death and disability worldwide". The investigators collated and analysed a prodigious quantity of data and modelled the numbers of years lived with disability (YLDs), years of life lost (YLLs), and deaths and disability-adjusted life years (DALYsthe combination of YLDs and YLLs) for a comprehensive range of ages and countries.

In 2010, four of the five leading risk factors for disease-high blood pressure, tobacco smoking including second-hand smoke, alcohol use, and diets low in fruitswere recognized causes of adult chronic disease, particularly cardiovascular diseases. These risk factors were estimated to be responsible for $7.0 \%, 6.3 \%, 5.5 \%$, and $4.2 \%$ of global DALYs, respectively. By contrast, in 1990, high blood pressure ranked as the fourth-highest cause of DALYs, behind being underweight during childhood, household air pollution, and smoking.

The rise in cardiovascular risk factors between 1990 and 2010 is indicative of a global epidemiological transition, but masks important regional disparities. In general, high blood pressure, tobacco smoking, alcohol use, and elevated BMI ranked highly as risk factors throughout North and South America, Europe, and the Asia-Pacific, but being underweight during childhood, household air pollution from solid fuels, suboptimal breast feeding, and iron deficiency remained leading causes of disease in most regions of Africa.

Globally, 52.8 million deaths were recorded in 2010. As in 1990, the two

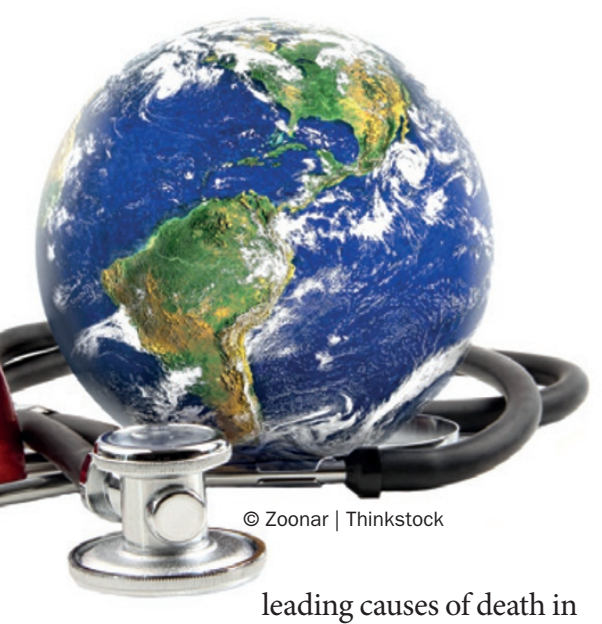

2010 were ischaemic heart disease $(13.3 \%)$ and stroke (11.1\%), but the proportion of deaths for which these conditions were responsible had increased from one-fifth to one-quarter over the 2 decades. The number of global YLLs from ischaemic heart disease and stroke increased by $28 \%$ and $17 \%$, respectively, over the 20 -year period, resulting in these conditions becoming the first and third causes of YLLs. Deaths attributed to atrial fibrillation or flutter increased by $233.9 \%$.

Again, the data reflect a global transition in diseases, with important regional heterogeneity. Substantial mortality from infectious diseases (diarrhoeal illnesses, HIV/AIDS, and malaria) persists in most of sub-Saharan Africa, compared with a predominance of cardiovascular diseases and cancers elsewhere. In the Middle East and North Africa, reports Dr Nizal Sarrafzadegan from Isfahan, Iran, "there is a big transition from communicable to noncommunicable diseases. Cardiovascular diseases are [now] the main reason for mortality and morbidity ... in this region."

The researchers also estimated the YLDs as a consequence of 1,160 sequelae from 289 diseases. The main contributors to global YLDs were mental and behavioural, musculoskeletal, and diabetic or endocrine disorders. Cardiovascular and circulatory diseases accounted for only $2.8 \%$ of all YLDs, but their importance increased progressively with age. The overall burden was similar around the world, but regional disparity in the underlying disease might exist. For example, 37.7 million cases of heart failure were associated with 4.2 million YLDs; however, ischaemic heart disease and chronic obstructive pulmonary disease were leading causes of YLDs in developed regions, whereas hypertensive heart disease, rheumatic heart disease, cardiomyopathy, and myocarditis were larger contributors in some developing areas, especially in Africa.

In a fourth paper, the investigators identified ischaemic heart disease as the leading cause of global DALYs in 2012, an increase of 29\% from fourth rank in 1990. Stroke increased by $19 \%$ over this period, and was the third-leading cause of DALYs in 2010. Of the 2.49 billion DALYs, 54\% were attributable to noncommunicable diseases, $11.8 \%$ of which resulted from cardiovascular disease (5.2\% ischaemic heart disease, $2.5 \%$ haemorrhagic stroke, $1.6 \%$ ischaemic stroke, and $0.6 \%$ hypertensive heart disease).

The Global Burden of Disease Study 2010 provides a plethora of epidemiological data. "Public policy to improve the health of populations will be more effective if it addresses the major causes of disease burden," conclude the researchers. "Even small reductions of ... exposure to large risks will yield substantial health gains."

Gregory B. Lim

Original articles Lim, S. S. et al. A comparative risk assessment of burden of disease and injury attributable to 67 risk factors and risk factor clusters in 21 regions, 1990-2010: a systematic analysis for the Global Burden of Disease Study 2010. Lancet 380, 2224-2260 (2012) Lozano, R. L. et al. Global and regional mortality from 235 causes of death for 20 age groups in 1990 and 2010: a systematic analysis for the Global Burden of Disease Study 2010. Lancet 380, 2095-2128 (2012) | Vos, T. et al. Years lived with disability (YLDs) for 1160 sequelae of 289 diseases and injuries 1990-2010: a systematic analysis for the Global Burden of Disease Study 2010. Lancet 380, 2163-2196 (2012) | Murray, C. J. L. et al. Disability-adjusted life years (DALYs) for 291 diseases and injuries in 21 regions, 1990-2010: a systematic analysis for the Global Burden of Disease Study 2010. Lancet 380 2197-2223 (2012) 